\title{
Inside out: unveiling local interstellar spectra of cosmic ray species
}

\author{
M. J. Boschini, ${ }^{a b}$ S. Della Torre, ${ }^{a}$ M. Gervasi, ${ }^{a c}$ D. Grandi, ${ }^{a}$ G. Jóhannesson, ${ }^{d}$ \\ M. Kachelrieß, ${ }^{e}$ G. La Vacca, ${ }^{a}$ N. Masi,${ }^{f}$ I. V. Moskalenko ${ }^{*},{ }^{g}$ E. Orlando, ${ }^{g}$ \\ S. S. Ostapchenko, ${ }^{h}$ S. Pensotti, ${ }^{a c}$ T. A. Porter,${ }^{g}$ L. Quadrani, ${ }^{f i}$ P. G. Rancoita, ${ }^{a}$ \\ D. Rozza ${ }^{a c}$ and M. Tacconi ${ }^{a c}$
}

${ }^{a}$ INFN, Milano-Bicocca, Milano, Italy

${ }^{b}$ CINECA, Segrate, Milano, Italy

${ }^{c}$ Physics Department, University of Milano-Bicocca, Milano, Italy

${ }^{d}$ Science Institute, University of Iceland, Reykjavik, Iceland and NORDITA, Stockholm, Sweden

${ }^{e}$ Institutt for fysikk, NTNU, 7491 Trondheim, Norway

${ }^{f}$ INFN, Bologna, Italy

${ }^{g}$ Hansen Experimental Physics Laboratory and KIPAC, Stanford University, Stanford, U.S.A.

${ }^{h}$ Frankfurt Institute of Advanced Studies, Frankfurt, Germany and Skobeltsyn Institute of Nuclear

Physics, Moscow State University, Moscow, Russia

${ }^{i}$ Physics Department, University of Bologna, Bologna, Italy

E-mail: matteo.boschini@mib.infn.it, Stefano.DellaTorre@mib.infn.it,

Massimo.Gervasi@mib.infn.it, davide.grandi@mib.infn.it,

gudlaugu@hi.is, michael.kachelriess@ntnu.no,

giuseppe. lavacca@mib.infn.it, nicolo.masi@bo.infn.it,

imosestanford.edu, eorlando@stanford.edu, sergei@tf.phys.ntnu.no,

simonetta.pensotti@mib.infn.it, tporterestanford.edu,

lucio.quadrani@cern.ch, piergiorgio.rancoita@unimib.it,

Davide.Rozza@mib.infn.it, mauro.tacconi@mib.infn.it

Local interstellar spectra (LIS) for protons, helium and antiprotons are built using the most recent experimental results combined with state-of-the-art models for propagation in the Galaxy and Heliosphere. Two propagation packages, GALPROP and HelMod, are combined to provide a single framework that is run to reproduce direct measurements of cosmic ray (CR) species at different modulation levels and at both polarities of the solar magnetic field. To do so in a selfconsistent way, an iterative procedure was developed, where the GALPROP LIS output is fed into HelMod that provides modulated spectra for specific time periods of selected experiments to compare with the data. The parameters were tuned with a maximum likelihood procedure using an extensive data set of proton spectra from 1997-2015. The proposed LIS accommodate both the low energy interstellar CR spectra measured by Voyager 1 and the high energy observations by BESS, PAMELA, AMS-01, and AMS-02 made from the balloons and near-Earth payloads. The proton LIS also accounts for Ulysses counting rate features measured out of the ecliptic plane. The obtained solution is in a good agreement with proton, helium, and antiproton data by AMS-02, BESS, and PAMELA in the whole energy range.

35th International Cosmic Ray Conference

10-20 July, 2017

Bexco, Busan, Korea 


\section{Introduction}

The launch of PAMELA [1] in 2006, followed by the Fermi Large Area Telescope (FermiLAT) [2] in 2008, and the AMS-02 [3] in 2011 signify the beginning of a new era in astrophysics. New materials and technologies employed by these space missions have enabled measurements with unmatched precision, which allows for searches of subtle signatures of new phenomena in CR and $\gamma$-ray data. These advances are built on solid results of earlier missions, such as ACE/CRIS, SuperTIGER, ATIC, BESS, CAPRICE, CREAM, HEAO-3, HEAT, ISOMAX, TIGER, TRACER, Ulysses, and many others. Launched in 1977 Voyager 1, 2 spacecrafts are providing unique data on the elemental spectra and composition at the interstellar reaches of the Solar system [36,37]. Other high-expectations missions are recently launched (CALET and DAMPE) or are awaiting for launch (ISS-CREAM). Indirect CR measurements are made through observations of their emissions by space- and ground-based telescopes: INTEGRAL, HAWC, H.E.S.S., MAGIC, VERITAS, WMAP, and Planck. The most spectacular is the Fermi-LAT mission that is mapping the all-sky diffuse $\gamma$-ray emission, produced by CR interactions in the interstellar medium, and near CR accelerators.

Our understanding of the origin of CRs, their acceleration mechanisms, main features of the interstellar propagation, and CR source composition comes from a combination of observational data and a strong theoretical effort [4]. Interpretation of many different kinds of data with a selfconsistent model requires a state-of-the-art numerical tool that combines the latest information on the Galactic structure (distributions of gas, dust, radiation and magnetic fields) with the latest formalisms describing particle and nuclear cross sections and description of the processes in the ISM. This was realized about 20 years ago, when some of us started to develop the most advanced fully numerical CR propagation code, called GALPROP ${ }^{1}[5,6]$. The key idea behind GALPROP is that all CR-related data, including direct measurements, $\gamma$-rays, synchrotron radiation, etc., are subject to the same Galactic physics and must be modeled simultaneously. The GALPROP model for CR propagation is being continuously developed in order to provide a framework for studies of CR propagation in the Galaxy and interpretation of relevant observations [4-16]. The latest version and supplementary datasets are available through a WebRun interface at the dedicated website.

Meanwhile, an accurate description of propagation of $\mathrm{CR}$ particles through the Heliosphere in the last $\sim 130 \mathrm{AU}$, that is a minuscule distance by the Galactic scale, was a considerable challenge until now. These last $0.0006 \mathrm{pc}$ are so important because they provide a link between the predictions of the interstellar propagation models with the location where almost all direct CR measurements are made. Even though, the heliospheric modulation affects only particles with energies below $30-50 \mathrm{GeV}$, this range includes the sub-GeV energies where the most precise measurements of CR isotopic composition are made. These low energy data are used to derive the parameters of interstellar propagation that are then extrapolated onto the whole Galaxy and all energies up to the multi- $\mathrm{TeV}$ region. Therefore, an improvement in the description of the heliospheric propagation has a global impact on our understanding of CR phenomena. However, in most applications the effect of solar modulation was treated using the simplest force-field approximation $[17,18]$ in which the diffusion tensor is approximated by a scalar and the resulting modulation effects are expressed with a spherically symmetric modulated differential number density. This was "matched" by the

\footnotetext{
* Speaker.

${ }^{1}$ http://galprop.stanford.edu
} 
uniform Leaky-Box model for Galactic propagation - a combination, which dominated the CR interpretation landscape in the second part of the 20th century and in the beginning of the 21st. With the development of sophisticated Galactic propagation models, the force-field approximation became the Achilles' heel of CR astrophysics. More advanced models did exist - including those accounting for the so-called "charge drift effect" [19-22], - but they were not as "user friendly" and required a high level of expertise in the heliospheric physics.

We use a recently developed version of a 2D Monte Carlo code for heliospheric propagation HelMod $^{2}$ [23-25] combined with GALPROP to take advantage of a significant progress in CR measurements to derive the LIS for protons, helium and antiprotons. The HelMod model includes all relevant effects and, thus, a full description of the diffusion tensor. It allows an accurate calculation of the heliospheric propagation for particles with rigidities $>1 \mathrm{GV}$. For more detail see [26].

\section{Calculations and results}

In order to derive the physically motivated LIS of CR species, an iterative procedure was developed to feed the GALPROP output into HelMod that provides modulated spectra for specific time periods to compare with AMS-02 data as observational constraints. The Markov Chain Monte Carlo (MCMC) interface to the development version of GALPROP was adapted from CosRayMC [28] and, in general, from COSMOMC package. Main propagation parameters were left free in the scan using the 2D GALPROP model (Table 1): the Galactic halo half-width $z$, the normalization of the diffusion coefficient $D_{0}$ at the reference rigidity $R_{D}=4.5 \mathrm{GV}$ and the index of its rigidity dependence $\delta$, the Alfvén velocity $V_{\text {Alf }}$, the convection velocity and its gradient ( $V_{\text {conv }}$, $d V_{\text {conv }} / d z$ ). The radial size of the Galaxy was set to $20 \mathrm{kpc}$. To correctly reproduce the AMS-02 proton data at low energies we introduced a factor $\beta^{\eta}$ in the diffusion coefficient, where $\beta=v / c$, and the best fit value of $\eta=0.91$. Parameters of the injection spectra, such as spectral indices and the break rigidities, were also left free, but their exact values depend on the solar modulation, so the low energy parts of the spectra are tuned together with the solar modulation parameters. The numerical values of the $\mathrm{CR}$ source distribution parameters [14], $z_{\text {scale }}=0.2, \alpha=1.5$, and $\beta=3.5$, remain unchanged for all scans.

A model with both reacceleration and convection was used. The solar modulation is calculated using numerical functions based on HelMod. In the scan we use all published AMS-02 data on protons [29], helium [30], B/C ratio [31] and electrons [32], while antiproton data were explicitly excluded - one of our goals is to make a prediction of the antiproton spectrum based on other CR data. The goodness estimator of the parameter scan is the natural logarithm of the likelihood function. The best fit values along with error estimates and the initial ranges are given in Table 1.

The combined convection-reacceleration model has a uniform spatial diffusion coefficient $\left(D_{0 x}=D_{0 z}\right)$ with a single power-law index $\left(\delta_{1}=\delta_{2}\right)$ in the whole rigidity range. The index $\delta$ of the rigidity dependence of the diffusion coefficient is derived from the slope of the secondaryto-primary ratio (e.g., B/C). A fit to the AMS- 02 measurements of the $\mathrm{B} / \mathrm{C}$ ratio [31] yields 0.395 , which is very close to the value $\delta=0.397 \pm 0.007$ found from the fit to the PAMELA data [34] and quite close to the Kolmogorov index of $1 / 3$. The MCMC procedure prefers a medium size halo of $4 \mathrm{kpc}$, also favored in the past studies [35].

\footnotetext{
${ }^{2}$ http://www.helmod.org/
} 


\begin{tabular}{rrcrrr} 
Parameter & Best Value & Units & $1 \sigma$ Mean Error & $\%$ Error & Scan Range \\
\hline \hline$z$ & 4.0 & $\mathrm{kpc}$ & 0.7 & 18 & {$[1-10]$} \\
$D_{0} / 10^{28}$ & 4.3 & $\mathrm{~cm}^{2} \mathrm{~s}^{-1}$ & 0.5 & 12 & {$[1-10]$} \\
$\delta$ & 0.395 & - & 0.025 & 6 & {$[0.3-0.9]$} \\
$V_{\text {Alf }}$ & 28.6 & $\mathrm{~km} \mathrm{~s}^{-1}$ & 3.0 & 10 & {$[0-40]$} \\
$V_{\text {conv }}$ & 12.4 & $\mathrm{~km} \mathrm{~s}^{-1}$ & 0.8 & 6 & {$[0-20]$} \\
$d V_{\text {conv }} / d z$ & 10.2 & $\mathrm{~km} \mathrm{~s}^{-1} \mathrm{kpc}^{-1}$ & 0.7 & 7 & {$[0-20]$} \\
\hline
\end{tabular}

Table 1: Propagation parameters values, obtained with the MCMC posterior distributions

\begin{tabular}{crrrrr} 
Parameters & $p$ & $\mathrm{He}$ & $e^{-}$ & Mean Error & Range \\
\hline \hline$R_{1}$ & $7 \mathrm{GV}$ & $7 \mathrm{GV}$ & $6 \mathrm{GV}$ & $1 \mathrm{GV}$ & {$[4-10]$} \\
$R_{2}$ & $360 \mathrm{GV}$ & $330 \mathrm{GV}$ & $100 \mathrm{GV}$ & $10 \mathrm{GV}$ & {$[300-400]$} \\
$\gamma_{1}$ & 1.69 & 1.71 & 1.45 & 0.06 & {$[1.5-2.1]$} \\
$\gamma_{2}$ & 2.44 & 2.38 & 2.75 & 0.04 & {$[2.1-2.7]$} \\
$\gamma_{3}$ & 2.28 & 2.21 & 2.49 & 0.05 & {$[2-2.4]$} \\
\hline
\end{tabular}

Table 2: Spectral parameters for protons, helium, and electrons

Note that the current MCMC setup has several distinct differences from those usually employed in the literature. (i) In the current scan we use $p, \mathrm{He}, \mathrm{B} / \mathrm{C}$, and $e^{-}$data from AMS-02 experiment only, i.e. only data $>2 \mathrm{GV}$ are used. (ii) Both reacceleration and convection processes are included simultaneously. (iii) We do not use the force-field approximation. Instead, for the modulation calculations we use the HelMod routine. (iv) The MCMC procedure is used to find the best values and confidence limits for the interstellar propagation parameters and the injection spectra. The interstellar propagation was fixed after this step. (v) A grid of GALPROP models is built using small, within a few per cent, variations of the best fit parameter values found at the previous step. This model grid is used for a fine tuning of the heliospheric propagation.

The MCMC procedure is used only in the first step to define a consistent parameter space, then a fine-tuning of the model employing the HelMod Module was performed. Thus the best values in Table 1 are not necessarily the most probable values (MPV) obtained with the MCMC procedure, but the final values which come from the GALPROP-HelMod combined fine tuning. The injection spectrum parameters for each species, such as the indices $\gamma_{i}$ below and above the rigidity breaks $R_{i}$, have been moved together with solar modulation parameters within physical ranges in order to find best fit solutions for all the observables (Table 2).

As pointed out in [13], there could be a significant difference between the propagation parameters derived from the light isotopes ( $p, \bar{p}, \mathrm{He}$ ), and nuclei (boron to silicon). Our study does not show any evident discrepancies between the light isotopes $(p, \bar{p}, \mathrm{He})$, and the $\mathrm{B} / \mathrm{C}$ ratio (nuclei). This may be attributed to the differences in the setups as explained above and, in particular, by a more realistic description of heliospheric propagation used in the present analysis.

Combination of AMS-02 high precision data with the data taken by earlier missions (AMS01, BESS, PAMELA) at different epochs allows the same GALPROP/HelMod framework to be 

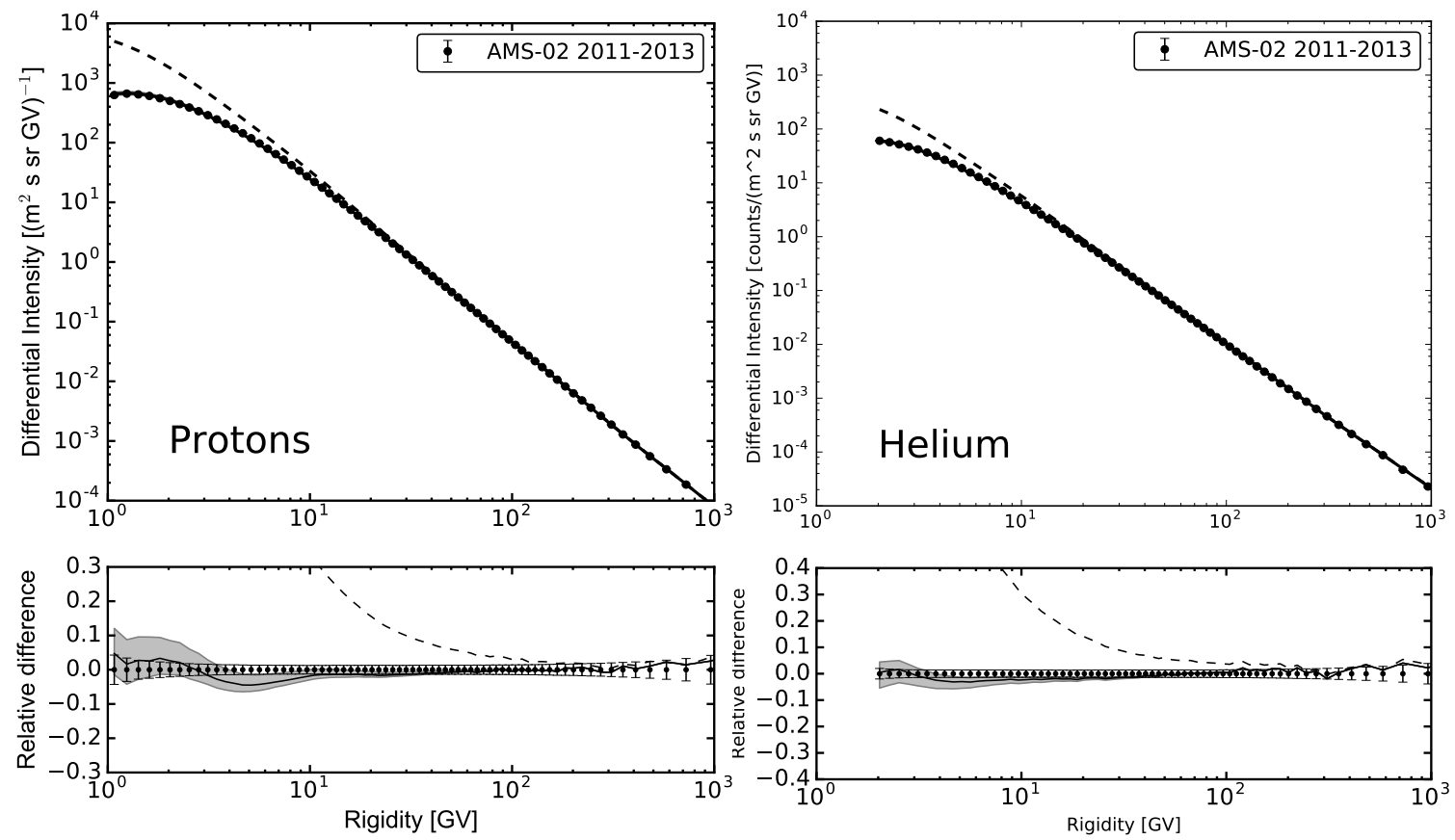

Figure 1: The differential intensities of CR protons (left) and helium (right) measured by AMS-02, dashed line is the GALPROP LIS, and solid line is the computed modulated spectrum. The bottom panel is the relative difference between the numerical solution and experimental data.

extended to account for different polarities of the solar magnetic field and for periods of high and low solar activity in cycles 23 and 24, while at the same time providing an accurate description of the Voyager 1 spectra taken outside of the Heliosphere. A detailed comparison made with observational data for conditions of low (i.e., 1997-1998, 2006-2010) and high solar activity (i.e. 2000-2002, 2011-2013), as well as with the moderate activity period, allows a unique model to be built that is valid for the entire solar cycle. Even though the data below $~ 200 \mathrm{MeV} / \mathrm{n}$ (ACE/CRIS, Voyager 1) were not included into the MCMC scan, the agreement between the model predictions and data is remarkable. Here we provide only illustrative results, more detail could be found in [26].

High solar activity periods are the most challenging from the viewpoint of theory of the heliospheric transport. Figure 1 shows the differential intensities of $\mathrm{CR}$ protons and helium as measured by the AMS-02 during the solar activity peak of cycle 24 [29,30], the GALPROP LIS, and the computed modulated spectrum. In Figure 2 (left) antiproton calculations are compared to the AMS-02 measurements corresponding to a period of high solar activity. The antiproton calculations include a contribution from CR nuclei through nickel. The GALPROP-HelMod spectrum appears slightly lower than the data in the range $\sim 7-20 \mathrm{GV}$, but the discrepancy does not exceed one standard deviation once the HelMod and AMS-02 errors are taken into account. More plots, tabulated spectra, and analytical fits to the derived LIS are given in paper [26].

The quality of the model description can be judged from a comparison with the Ulysses observations taken from 1990s through 2009 [38-40] outside the ecliptic plane up to $\pm 80^{\circ}$ in solar latitude and at distances $\sim 1-5$ AU from the Sun. We have shown $[24,41]$ that a combination of a polar modification in the description of the heliospheric magnetic field with a diffusion tensor that 

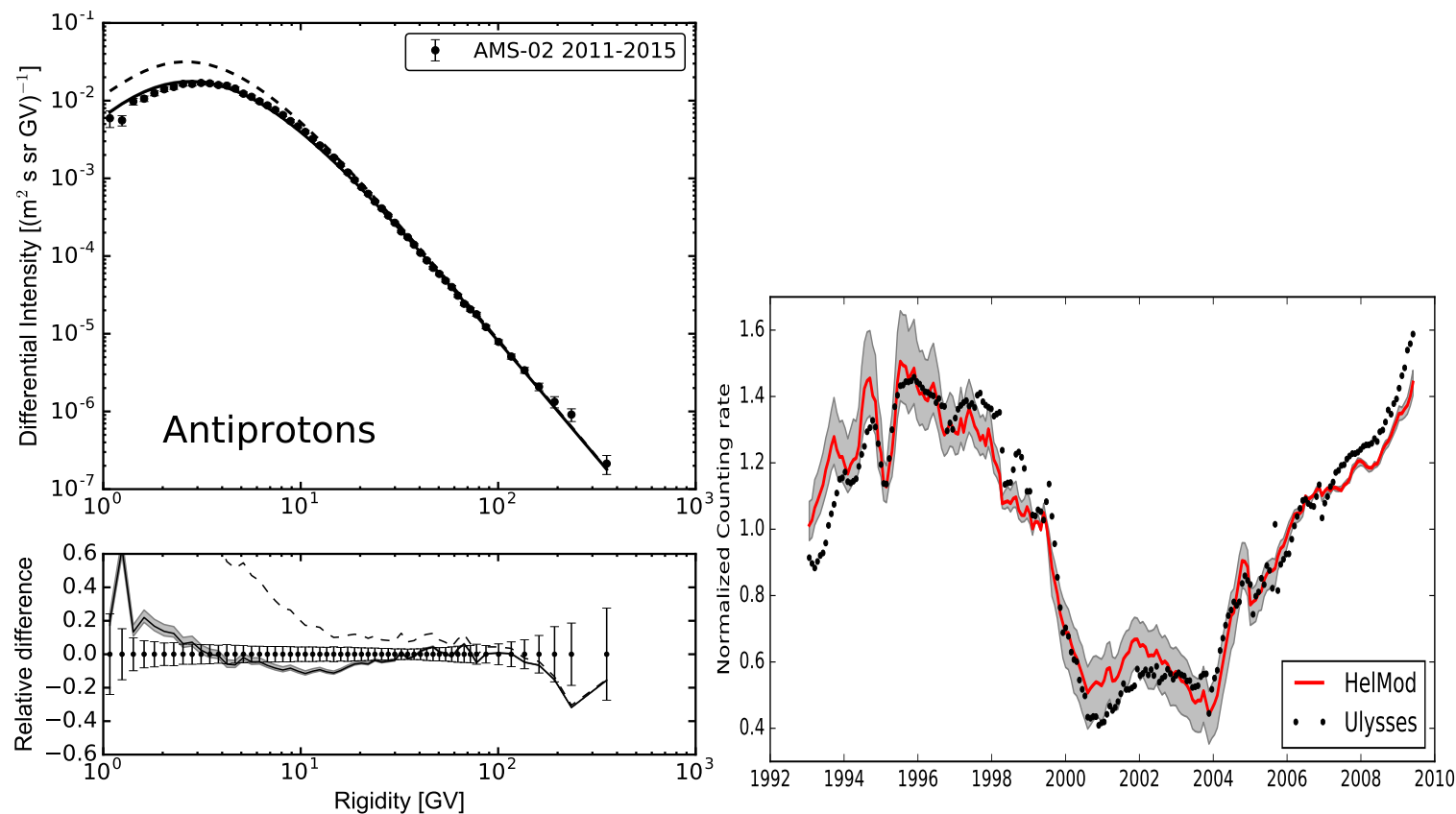

Figure 2: Left panel: The differential intensity of CR antiprotons measured by AMS-02, dashed line is the GALPROP LIS, and solid line is the computed modulated spectrum. The bottom panel is the relative difference between the model and experimental data. Right panel: Ulysses counting rate normalized to the average value for the KET coincidence channel K12 (proton energy $0.25-2.2 \mathrm{GeV}$ ) averaged over a Carrington rotation. Solid line - the HelMod calculations for protons of $2.2 \mathrm{GeV}$.

is independent on the solar latitude is able to reproduce the measured latitudinal gradients during the low solar activity periods. In Figure 2 (right) we compare the Ulysses normalized counting rate with the HelMod calculations.

To conclude, the proposed LIS provide good description of all the data (Voyager 1, BESS, PAMELA, AMS-01, and AMS-02) for solar cycles 23 and 24 in the whole energy range.

\section{Acknowledgments}

We thank P. Bobik, G. Boella, K. Kudela, M. Putis and M. Zannoni for their support to the HelMod code and many useful suggestions. This work is supported by ASI under contract ASI-INFN I/002/13/0 and by ESA through contract 4000116146/16/NL/HK. I.V.M., E.O., T.A.P. acknowledge support from NASA Grants Nos. NNX13AC47G, NNX17AB48G, NNX16AF27G, NNX15AU79G. S.S.O. is supported by Deutsche Forschungsgemeinschaft, project OS 481/1.

\section{References}

[1] P. Picozza, et al., PAMELA - A payload for antimatter matter exploration and light-nuclei astrophysics, Astropart. Phys. 27 (2007) 296.

[2] W. B. Atwood, et al., The Large Area Telescope on the Fermi Gamma-Ray Space Telescope Mission, ApJ 697 (2009) 1071. 
[3] M. Aguilar, et al., First Result from the Alpha Magnetic Spectrometer on the International Space Station: Precision Measurement of the Positron Fraction in Primary Cosmic Rays of 0.5-350 GeV, Phys. Rev. Lett. 110 (2013) 141102.

[4] A. W. Strong, et al., Cosmic-Ray Propagation and Interactions in the Galaxy, Annual Review of Nuclear and Particle Science 57 (2007) 285.

[5] I. V. Moskalenko, \& A. W. Strong, Production and Propagation of Cosmic-Ray Positrons and Electrons, ApJ 493 (1998) 694.

[6] A. W. Strong, \& I. V. Moskalenko, Propagation of Cosmic-Ray Nucleons in the Galaxy, ApJ 509 (1998) 212.

[7] I. V. Moskalenko, \& A. W. Strong, Anisotropic Inverse Compton Scattering in the Galaxy, ApJ 528 (2000) 357.

[8] A. W. Strong, et al., Diffuse Continuum Gamma Rays from the Galaxy, ApJ 537 (2000) 763.

[9] I. V. Moskalenko, et al., Secondary Antiprotons and Propagation of Cosmic Rays in the Galaxy and Heliosphere, ApJ 565 (2002) 280.

[10] I. V. Moskalenko, et al., Challenging Cosmic-Ray Propagation with Antiprotons: Evidence for a "Fresh” Nuclei Component?, ApJ 586 (2003) 1050.

[11] A. W. Strong, et al., Diffuse Galactic Continuum Gamma Rays: A Model Compatible with EGRET Data and Cosmic-Ray Measurements, ApJ 613 (2004) 962.

[12] V. S. Ptuskin, et al., Dissipation of Magnetohydrodynamic Waves on Energetic Particles: Impact on Interstellar Turbulence and Cosmic-Ray Transport, ApJ 642 (2006) 902.

[13] G. Jóhannesson, et al., Bayesian Analysis of Cosmic Ray Propagation: Evidence against Homogeneous Diffusion, ApJ 824 (2016) 16.

[14] R. Trotta, et al., Constraints on Cosmic-ray Propagation Models from A Global Bayesian Analysis, ApJ 729 (2011) 106.

[15] A. E. Vladimirov, et al., GALPROP WebRun: An internet-based service for calculating galactic cosmic ray propagation and associated photon emissions, Comp. Phys. Comm. 182 (2011) 1156.

[16] A. E. Vladimirov, et al., Testing the Origin of High-energy Cosmic Rays, ApJ 752 (2012) 68.

[17] L. J. Gleeson, \& W. I. Axford, Cosmic Rays in the Interplanetary Medium, ApJL 149 (1967) L115.

[18] L. J. Gleeson, \& W. I. Axford, Solar Modulation of Galactic Cosmic Rays, ApJ 154 (1968) 1011.

[19] M. Garcia-Munoz, et al., The dependence of solar modulation on the sign of the cosmic ray particle charge, JGR 91 (1986) 2858.

[20] G. Boella, et al., Evidence for charge drift modulation at intermediate solar activity from the flux variation of protons and $\alpha$ particles, JGR 106 (2001) 29355.

[21] J. R. Jokipii, et al., Effects of particle drift on cosmic-ray transport. I - General properties, application to solar modulation, ApJ 213 (1977) 861.

[22] R. A. Burger, Modeling Drift along the Heliospheric Wavy Neutral Sheet, ApJ 760 (2012) 60.

[23] P. Bobik, et al., Systematic Investigation of Solar Modulation of Galactic Protons for Solar Cycle 23 Using a Monte Carlo Approach with Particle Drift Effects and Latitudinal Dependence, ApJ $\mathbf{7 4 5}$ (2012) 132. 
[24] P. Bobik, et al., Latitudinal Dependence of Cosmic Rays Modulation at 1 AU and Interplanetary Magnetic Field Polar Correction, Adv. Astron. 2013 (2013) 793072.

[25] P. Bobik, et al., On the forward-backward-in-time approach for Monte Carlo solution of Parker's transport equation: One-dimensional case, JGR (Space Phys.) 121 (2016) 3920.

[26] M. J. Boschini, et al., Solution of Heliospheric Propagation: Unveiling the Local Interstellar Spectra of Cosmic-ray Species, ApJ $\mathbf{8 4 0}$ (2017) 115.

[27] P. Bobik, et al., Antiproton modulation in the Heliosphere and AMS-02 antiproton over proton ratio prediction, Astrophys. Spa. Sci. Trans. 7 (2011) 245.

[28] J. Liu, et al., Cosmic ray Monte Carlo: A global fitting method in studying the properties of the new sources of cosmic $e^{ \pm}$excesses, Phys. Rev. D 85 (2012) 043507.

[29] M. Aguilar, et al., Precision Measurement of the Proton Flux in Primary Cosmic Rays from Rigidity 1 GV to 1.8 TV with the Alpha Magnetic Spectrometer on the International Space Station, Phys. Rev. Lett. 114 (2015) 171103.

[30] M. Aguilar, et al., Precision Measurement of the Helium Flux in Primary Cosmic Rays of Rigidities 1.9 GV to 3 TV with the Alpha Magnetic Spectrometer on the International Space Station, Phys. Rev. Lett. 115 (2015) 211101.

[31] M. Aguilar, et al., Precision Measurement of the Boron to Carbon Flux Ratio in Cosmic Rays from 1.9 GV to 2.6 TV with the Alpha Magnetic Spectrometer on the International Space Station, Phys. Rev. Lett. 117 (2016) 231102.

[32] M. Aguilar, et al., Electron and Positron Fluxes in Primary Cosmic Rays Measured with the Alpha Magnetic Spectrometer on the International Space Station, Phys. Rev. Lett. 113 (2014) 121102.

[33] M. Aguilar, et al., Antiproton Flux, Antiproton-to-Proton Flux Ratio, and Properties of Elementary Particle Fluxes in Primary Cosmic Rays Measured with the Alpha Magnetic Spectrometer on the International Space Station, Phys. Rev. Lett. 117 (2016) 091103.

[34] O. Adriani, et al., Measurement of Boron and Carbon Fluxes in Cosmic Rays with the PAMELA Experiment, ApJ 791 (2014) 93.

[35] A. W. Strong, \& I. V. Moskalenko, Models for galactic cosmic-ray propagation, Adv. Space Res. 27 (2001) 717.

[36] A. C. Cummings, et al., Galactic Cosmic Rays in the Local Interstellar Medium: Voyager 1 Observations and Model Results, ApJ 831 (2016) 18.

[37] E. C. Stone, et al., Voyager 1 Observes Low-Energy Galactic Cosmic Rays in a Region Depleted of Heliospheric Ions, Science 341 (2013) 150.

[38] T. R. Sanderson, et al., High-Latitude Observations of Energetic Ions During the First Ulysses Polar Pass, Spa. Sci. Rev. 72 (1995) 291.

[39] R. G. Marsden, The 3-D Heliosphere at Solar Maximum, PASP 113 (2001) 129.

[40] A. Balogh, et al., The Ulysses perspective, in proc. of The Heliosphere near Solar Minimum, Springer, London 2001.

[41] P. Bobik, et al., Cosmic Ray Modulation studied with HelMod Monte Carlo tool and comparison with Ulysses Fast Scan Data during consecutive Solar Minima, (2013) [arXiv:1307.5199]. 